\title{
UNA NUEVA PERSPECTIVA DE LA EDAD DEL BRONCE
}

\author{
POR \\ J. M. VAZQUEZ VARELA ( $\left(^{\circ}\right)$ \\ J. A. CANO PAN (")
}

\begin{abstract}
RESUMEN La presencia de elementos típicos de la "Cultura de los cantos tallados» en el yacimiento del Bronce Final de Portecelo, Pontevedra, plantea problemas sobre la visión tradicional de la Edad del Bronce y de la "Cultura de los cantos tallados". Se propone la elaboración de un modelo interpretativo más amplio para esta época y un uso más crítico del término "Cultura de los cantos tallados".
\end{abstract}

\begin{abstract}
Based on information from excavations in the Late Bronze Age settlement of Portecelo in the North-West of Spain it is suggested a new perspective about the Bronze Age and the Pebble Culture.

Palabras clave: Edad del Bronce. Cultura de los cantos tallados. Bronce Atlántico. Revisión del modelo tradicional.
\end{abstract}

Se presentan unas breves reflexiones preliminares sobre el yacimiento de Portecelo, 0 Rosal, Pontevedra (Cano, 1988) perteneciente al Bronce Final, que ayudan a comprender la Edad del Bronce desde una perspectiva diferente.

El principal aspecto que retiene nuestra atención sobre este hábitat de un momento temprano del Bronce Final, de acuerdo con la tipología de la cerámica y una datación de C 14 es la ausencia de toda huella de uso del metal y la abundancia de la industria lítica obtenida a partir de cantos rodados recogidos en el litoral inmediato. La presencia de diferentes tipos de cantos tallados unifaciales y bifaciales, protodiscos, reaederas, denticulados, raspadores, escotaduras y "becs» en un yacimiento de cronología tan avanzada, nos lleva a reflexionar sobre la causa de la presencia de estas piezas, consideradas tradicionalmente como propias de períodos muy anteriores, en un momento tan avanzado de la Prehistoria. Igualmente atrae nuestra atención las posibles implicaciones que este hecho pueda tener para la visión tradicional de la Edad del Bronce y del «Complejo técnico de los cantos tallados".

Centrándonos en este último punto y para comprobar qué grado de validez puede tener este concepto hoy en día, se decidió comparar las colecciones líticas procedentes del yacimiento de

(") Departamento de Historia-1. F. Geografía e Historia. Universidad de Santiago de Compostela. Grupo de Estudios Prehistóricos «Padre Carballow. 
Portecelo, de los castros de A Forca y Santa Trega (Cano, 1988b) con las que se recuperaron en yacimientos del Paleolitico Inferior tales como el Aculadero, Budiño y Pinedo.

La comparación de estas colecciones se realizó con el fin de conocer las similitudes y diferencias existentes entre estos conjuntos que, estando tan separados en el tiempo y en el espacio, en los casos de el Aculadero y Pinedo y con bases económicas y sociales tan distintas, presentan una gama de artefactos líticos tallados que a priori resultan bastante semejantes.

Las piezas que sirven de base a este estudio proceden de los siguientes yacimientos:

El Aculadero (Cádiz): Se atribuye al Paleolítico Inferior dentro de la "Cultura de los cantos tallados" (Querol y Santonja, 1983).

Pinedo (Toledo): Se sitúa cronológicamente en el Paleolítico Inferior, en el Achelense antiguo (Querol y Santonja, 1979).

Budiño (Pontevedra): Este yacimiento, a pesar de la polémica suscitada sobre su cronología, se encuadra en el Paleolitico Inferior en el Achelense medio (Vidal, 1983).

Portecelo (Pontevedra): Pertenece a un momento temprano dentro del Bronce Final del Noroeste penínsular (Cano, 1988a).

Castro da Forca (Pontevedra): Es un castro dentro de la plena Edad del Hierro (Cano, 1987).

Castro de Santa Trega (Pontevedra): Cronológicamente se escapa de la Prehistoria ya que pertenece al mundo castreño romanizado en torno al cambio de Era (Peña, 1987; Cano, 1988c).

La realización del análisis comparativo encierra una serie de dificultades derivadas del empleo de distintas tipologías y metodologías en el estudio de estos conjuntos. La tipología que se utiliza en los tres yacimientos del Paleolítico Inferior es la desarrollada por Querol y Santonja (1978) para la clasificación de los cantos tallados basándose en criterios morfológicos y técnicos. Para los otros yacimientos se empleó un sistema tipológico confeccionado a partir de la tecnología y de la dinámica evolutiva de transformación de las piezas (Cano, 1988b). Para comparar los materiales de un modo homogéneo se aplicó el sistema de clasificación usado para los antiguos a los procedentes de los yacimientos modernos. Otra dificultad es de indole estadística. El análisis comparativo se efectúa en el caso de las colecciones antiguas a partir de la bibliografía con el consiguiente riesgo que esto entraña ya que en algunos casos, básicamente para Pinedo, algunos de los porcentajes que se presentan son aproximativos. De todos modos, pese a esta dificultad, los datos son válidos para el fin que aquí se persigue aunque somos conscientes de que se deberían revisar los materiales de todas las colecciones siguiendo la misma metodología para dejar sentenciado el problema de un modo definitivo.

La comparación en el caso de Budiño sólo se hace con los cantos tallados. En Pinedo no se tienen en cuenta los porcentajes de bifaces, hendedores y triedros ya que estos tipos, que aquí no superan el $4 \%$ del total de los objetos recogidos no aparecen en los otros yacimientos, salvo en Budiño, y lo que interesa es el estudio comparativo de los cantos tallados y los útiles sobre lasca dejando aparte los elementos característicos del grupo achelense.

COMPOSICION DE LOS CONJUNTOS LITICOS (\%)

\begin{tabular}{lccccr}
\hline & ACULADERO & PINBDO & PORTECELO & FORCA & TREGA \\
\hline Cantos tallados & 33,4 & 15,9 & 6,58 & 31,6 & 27,91 \\
Utiles sobre lasca & 20,5 & 8,0 & 4,99 & $\overline{7}$ & 2,50 \\
Núcleos & 4,7 & 4,0 & $\overline{7}$ & 0,73 & $\overline{7}$ \\
Lascas & 27,4 & 70,0 & 72,90 & 47,79 & 27,29 \\
Fragmentos & 14,0 & - & 15,51 & 19,85 & 42,29 \\
\hline
\end{tabular}

En la composición de la gama industrial, con las salvedades ya mencionadas, se aprecia que en el Aculadero y en los Castros, hay un porcentaje elevado de cantos tallados bastante similar. La 
ausencia de Núcleos en Portecelo, Trega y su poca importancia en el caso de Forca, se podría deber a que en ellos los cantos tallados cumplieron una doble función de artefactos y de núcleos, mientras que en los yacimientos antiguos habría una diferenciación mayor entre unos y otros. Pero después de revisar la interpretación gráfica de muchos de los núcleos del Aculadero y Pinedo, nos inclinamos a precisar que las diferencias se deben principalmente al método de estudio, ya que para nosotros una parte de los cantos dibujados serían artefactos y no núcleos.

No ocurre así con los útiles sobre lasca que están ausentes en A Forca y con el bajo porcentaje de lasca, que, si bien en el Aculadero está justificado, en los Castros hay que achacarlo a los métodos de excavación que se siguen para las etapas más avanzadas de la Prehistoria.

PORCENTAJES DE LAS COLECCIONES SEGUN LA FACIALIDAD, LA FORMA DEL FILO Y LA EXTENSION DE LA TALLA

\begin{tabular}{lcccrcr}
\hline & ACULADERO & PINEDO & BUDINo & $\begin{array}{r}\text { POR- } \\
\text { TECELO }\end{array}$ & FORCA & TREGA \\
\hline Unifaciales & 76,8 & 69,8 & 61,42 & 91,66 & $\mathbf{7 3 , 8 0}$ & $\mathbf{7 5 , 3 9}$ \\
Bifaciales & 23,2 & 30,2 & 38,50 & 8,33 & 26,19 & 23,80 \\
C. con filo simple & 86,2 & 69,5 & 81,69 & 75,00 & 59,52 & 69,84 \\
C. con filo convergente & 11,3 & 27,5 & 16,90 & 21,60 & 11,90 & 11,11 \\
C. con filo doble & 2,5 & 3,0 & 1,40 & 3,33 & 28,57 & 19,04 \\
Talla en menos 1/2 anverso & 82,7 & 70,7 & 70,00 & 33,88 & 69,04 & 73,01 \\
Talla en más 1/2 anverso & 17,3 & 29,3 & 30,00 & 66,02 & 30,95 & 26,98 \\
\hline
\end{tabular}

En este cuadro, en el que aparecen las características de los cantos tallados, se aprecia en lo referente a la Facialidad, un predominio de los Unifaciales sobre los Bifaciales, en todos los yacimientos, especialmente en Portecelo.

El abultado predominio en este yacimiento de los Unifaciales contrasta con la penetración de los desprendimientos que aquí ocupan más de $1 / 2$ anverso en la mayoría de los casos, mientras que en el resto es al contrario.

En lo que se refiere al primer nivel de diferenciación de la clasificación de Querol y Santonja (1978) se puede observar que en todas las colecciones hay un dominio de los cantos con filo simple seguido de los cantos con filo Convergente en Aculadero, Pinedo, Budiño y Portecelo y, por último, los cantos con filo Doble. En los Castros se invierten estas posiciones, ocupando los Dobles el segundo lugar.

\section{REPRESENTACION DE LOS UTILES SOBRE LASCA (\%)}

\begin{tabular}{lcrrr}
\hline & ACULADERO & PINEDO & PORTECELO & TREGA \\
\hline Raederas & 7,09 & 38,61 & 12,34 & 12,5 \\
Raspadores & 1,21 & 1,58 & 1,23 & - \\
Buriles & 0,17 & 0,59 & 1,23 & $4, \overline{7}$ \\
Muescas & 18,33 & 6,53 & 6,17 & 4,16 \\
Denticulados & 3,46 & 3,96 & 23,45 & 5,46 \\
Bec y perforadores & 5,53 & 10,89 & 41,97 & 50,00 \\
Lascas retocadas & 53,28 & 26,93 & 11,11 & 29,16 \\
Otros & 10,84 & & \\
\hline
\end{tabular}

En este cuadro, en el que aparecen los útiles sobre lasca, vemos que entre los seis tipos y las lascas retocadas suman más del $70 \%$ del conjunto. El apartado de otros lo engloban, en el caso del 
Aculadero y Pinedo, las lascas seudolevallois y otros tipos y en Portecelo y Santa Trega lo que nosotros denominamos útiles sobre lasca, con morfologia de artefactos nucleares (Cano, 1988b).

Las más abundantes son, con la excepción de Pinedo en el que las raederas son el $38,61 \%$, las lascas retocadas, que en Portecelo, Aculadero y Trega tienen porcentajes parecidos. A éstos le siguen las raederas, muescas y denticulados con cierta importancia, al igual que los "bec" y perforadores que sobre todo destacan en el Aculadero.

En el Castro de Santa Trega se aprecia un descenso cuantitativo importante de estas piezas y en Portecelo no se recogió ninguna, aunque algunas de las lascas podrían pasar por raederas, si no fuera que sus desprendimientos se deben al empleo del filo de esas lascas.

La mayor parte de las lascas de todos los yacimientos tienen superficies corticales como corresponden a las que se extraen de cantos rodados.

El retoque predominante es, en todas las colecciones, el Simple.

Lo que se desprende a "groso modo" de los cuadros y datos que se presentan, que son provisionales, es que las industrias líticas y fundamentalmente los cantos tallados que proceden de estos yacimientos, tienen más semejanzas que diferencias. De hecho las colecciones de los tres yacimientos recientes son más próximas al Aculadero que las del propio Pinedo o Budiño ya que las industrias de Portecelo, A Forca y Santa Trega tienen un conjunto de características (alto porcentaje de cantos tallados, ausencia de bifaces, hendedores, triedros y técnica levallois y la presencia de ciertos útiles sobre lasca) que son las que individualizan al Aculadero y constituyen el contenido esencial de la llamada «Cultura de los cantos tallados".

De hecho, si se prescindiera del contexto en que aparecen las colecciones de los yacimientos castreños y del Bronce y se estudiaran aisladamente es muy posible que se considerasen pertenecientes a una industria arcaica encuadrable en momentos muy antiguos de la etapa Paleolítica.

Por todo lo expuesto, y dado que las características tipológicas y técnicas que se consideran específicas del complejo técnico de los cantos tallados aparecen en contextos tan diversos como las comunidades de cazadores-recolectores del Pleistoceno Inferior, Metalúrgicos del final de la Edad del Bronce o comerciantes de la cultura castreña en época romana, no tiene sentido el empleo del concepto ni en sentido estricto, tal como se ha venido usando por la mayoría de los investigadores para referirse a las industrias más antiguas ni en sentido lato, para designar cualquier conjunto de cantos tallados de épocas más recientes. La aparición de una industria lítica de este tipo en un yacimiento del Bronce Final no deja de llamar poderosamente la atención sobre todo cuando advertimos la ausencia total en Portecelo de cualquier producto metálico, cosa que no ocurre en los castros citados de cronología más avanzada. En aquél no sólo no está presente ningún resto indicativo de la actividad metalúrgica sino que no se ha encontrado ningún desperdicio de metal. La ausencia de éste contrasta con la abundancia de la industria lítica, cuya perfecta caracterización nos indica que no es un producto de fortuna debido a un empleo esporádico de la piedra con una finalidad episódica sino que, por el contrario, es la firme señal de una constante talla realizada por expertos que utilizan la piedra de un modo habitual con un gran conocimiento de las propiedades de los diferentes materiales y un buen dominio de la técnica adecuada. Las características citadas ayudan a comprender la ausencia del metal. Si la piedra ha sido usada de un modo reiterado y magistral esto se debe probablemente a que la falta de metal en el yacimiento era crónica. Ya que en arqueología la ausencia de una prueba no es prueba de su ausencia, cabe considerar que el metal podría haber sido usado en Portecelo, pero dado su gran valor todo resto del mismo, en el momento de su ocupación o con posterioridad, habría sido aprovechado para reciclarlo. Esta posibilidad no se ve avalada por el uso cotidiano y experto de la piedra tallada que por el contrario sugiere que aquél fue escaso o prácticamente inexistente en el yacimiento, pues de lo contrario no hubiese sido necesaria una actividad explotadora de las posibilidades de la piedra tan elevada como se realizó en Portecelo que es mayor que en los castros citados, A Forca y Santa Trega, donde el metal está presente.

$\mathrm{Si}$ aceptamos las razones expuestas que indican una gran explotación de la piedra frente a la ausencia de metal, se plantea un fenómeno muy curioso tal cual es el de la existencia de un hábitat 
del Bronce Final en el noroeste de la Peninsula Ibérica, donde el metal brilla por su ausencia. Es chocante que precisamente en esta zona de la Peninsula, punto clave dentro del mundo del Bronce Atlántico y de las relaciones atlánticas que para esta época alcanzan un apogeo notable, halla un hábitat donde no sólo no aparece el metal, sino que como sustituto del mismo se emplea la talla de la piedra con gran destreza continuando con un tipo de artefactos y una cadena de gestos técnicos que tiene una larga tradición, tanto en ésta como en otras áreas del planeta.

El contraste señalado, un equipo técnico de piedra tallada que sigue una larga tradición, constituye un "arcaísmo", a reservas del tratamiento que este término requiere, en el Bronce Final de una de las zonas paradigmáticas de esta época en el Atlántico europeo. Este contraste se presta a múltiples lecturas independientemente de cuáles sean sus causas. Una de las reflexiones que se desprende a primera vista, es la contradicción entre las enseñanzas de Portecelo y las normas canónicas que definen al Bronce Final en la fachada atlántica europea en general y al noroeste de la Península Ibérica en particular. En la visión tradicional canónica la dorada o la bella edad del Bronce, como gusta de definirla J. Briard (1985) se viene caracterizando por una sociedad dinámica, en la que sobre un sector agricola, ganadero y explotador de los recursos marinos se desarrolla una intensa actividad de mineros, metalúrgicos, comerciantes y navegantes vinculados con la producción y difusión del metal, fundamentalmente el bronce, con una aleación muy equilibrada, señal de la gran habilidad de los técnicos de los talleres de fundición. Sobre este mundo de técnicos y comerciantes habría el desarrollo de jefes guerreros, parte de cuya riqueza derivaría del control de la circulación del metal. Este circularía de un modo abundante no sólo dentro de los territorios de cada comunidad, sino entre comunidades próximas e incluso lejanas. Uno de los paradigmas de este "tráfico" entre países distantes sería el de las relaciones atlánticas en las que precisamente Galicia, y de un modo especial la costa gallega, jugaría un papel de pivote importante entre la fachada occidental de la Península Ibérica y los otros finisterres atlánticos.

En modo alguno vamos a rechazar aquí esta construcción ampliamente aceptada, sino que tan sólo señalaremos algunas cuestiones de concepto y de método que pueden ayudar a comprender mejor la realidad de la Edad del Bronce en general y del Bronce Final en particular.

En Portecelo nada de la visión canónica anteriormente sintetizada de la Edad del Bronce aparece reflejado, sino todo lo contrario. En principio en este esquema no parecen tener lugar unos "arcaicos" o «bárbaros" talladores de las piedras de la costa que no tienen huellas de haber disfrutado de las delicias del metal. Estos «bárbaros" que tallaban la piedra siguiendo modos millonarios o al menos multimilenarios en años y recolectaban bellotas son, en realidad, contrarios a los principios generalmente admitidos de la sociedad de la Protohistoria de Europa, y constituyen "la otra cara de la Edad del Broncen.

Si la datación de Portecelo es correcta y la interpretación de sus materiales y estructuras válidas, entonces caben dos posibilidades: la primera, que el yacimiento sea una excepción que no haría sino confirmar la regla que en este caso sería el paradigma descrito anteriormente sobre la Edad del Bronce, con lo cual el único problema que plantearía el yacimiento sería el de explicar las causas de su anomalía. La segunda posibilidad es considerar que hay problemas en la visión canónica del Bronce Final. Dado que Portecelo no es un «unicum», ya que existe no muy lejos de él al menos otro yacimiento similar y que en otros puntos de las Rías Bajas Gallegas se han localizado asentamientos arqueológicos con características parecidas, tenemos que optar por la última posibilidad indicada que señala que algo no funciona bien en el modelo general descriptivo e interpretativo de la Edad del Bronce. Sobre esta opción se prosigue el análisis, pues no cabe duda de que existen fallos en la visión global de esta época que a nuestro entender derivan de una aplicación no demasiado feliz de la metodología de estudio. La escasez de necrópolis y de hábitats de este momento, muy grande para la fachada atlántica peninsular y mucho más notoria para el noroeste de la misma, ha reducido de un modo notable las posibilidades de estudio, que se han centrado de un modo fundamental en los productos metálicos procedentes en su mayoría de depósitos o de hallazgos aislados. Estos han constituido la base fundamental cuando no la única para documentar las culturas de la Edad del Bronce. Otras manifestaciones ergológicas contemporáneas permanecen 
todavía en un estadio preliminar de la investigación, como por ejemplo la cerámica y la piedra tallada y otras se estudian de un modo descontextualizado consideradas como un dominio autónomo, tal como sucede en el caso de los petroglifos. Esta desigualdad de las fuentes han hecho que dominen los estudios tipológicos $\mathbf{y}$ en menor medida arqueometalúrgicos sobre los productos metálicos del momento. Es sobre estos estudios en los que se ha basado la periodización, la cronología y las teorias sobre la sociedad en el Bronce.

Esta visión, basada en el metal como guia del periodo, en el mejor de los casos es parcial y por tanto sesgada e insuficiente. Para obtener una panorámica global más rigurosa de este mundo es necesario completarla con otras fuentes y utilizar éstas como contraste de los modelos derivados del análisis exclusivo del metal. Una investigación más comprensiva de todos los aspectos de la Edad del Bronce debiera haberse hecho antes de lanzarse a aventuradas construcciones basadas en elementos parciales. Este defecto de hacer un montaje teórico sobre una época basándose en un único aspecto del material arqueológico no es exclusivo de la Edad del Bronce, sino que es una peculiaridad de los estudios de Prehistoria y Protohistoria en los que para cada época se enfocan sólo sobre aspectos muy concretos de los materiales arqueológicos, en ocasiones, como ocurre en el Paleolítico Inferior y Medio, el predominio de la piedra es tal que no queda otro remedio, aunque esto no justifica el estado de abandono en que han permanecido los restos de huesos trabajados de estas dilatadas épocas. Para el Paleolítico Superior ya existe un equilibrio entre los estudios sobre la industria lítica y la ósea, en el periodo siguiente vuelve a predominar el estudio de la piedra. En el Neolítico va a ser la cerámica la que lleve la voz cantante en cuanto al mayor número de estudios y a un empleo para establecer culturas, periodizaciones, cronologías, etc. Con el advenimiento y generalización del uso del metal será éste el que reemplace a la cerámica como base de las investigaciones del período. Está claro que cuando no hay más que un tipo de material o de los varios conservados sólo uno de ellos es significativo, como es el caso de la piedra en el Paleolítico más antiguo, no hay más remedio que emplearla como base pero sin olvidar que se maneja una parte de la realidad y que, por tanto, el resultado final va a ser incompleto.

Lo que tiene menos sentido es el seguir con el mismo criterio en épocas en las que disponemos de diferentes tipos de fuentes como es en el caso que nos ocupa donde al lado de la metalurgia hay industria cerámica, ósea, lítica y lígnica de gran interés que ha sido olvidada más allá de lo cientificamente deseable. Esta actitud de enfoque sectorial de los estudios sobre una parcela de la realidad ha de ser corregida porque metodológicamente es incorrecta y ha llevado a reconstrucciones incompletas del pasado motivadas quizá en última instancia porque aquellos que hacen arqueología prehistórica o protohistórica son en realidad arqueólogos a los que sólo interesa el estudio del objeto como un fin y no historiadores que más allá de la arqueología quieren hacer una reconstrucción del pasado humano y tratar de entender su dinámica.

Desde esta perspectiva, Portecelo o mejor la visión de la Edad del Bronce que obtenemos a partir de los datos del yacimiento y que aparentemente contradice el modelo teórico tradicional, es una seria llamada a la atención hacia la necesidad de estudios que abarquen a todos los materiales para obtener modelos de validez general. La visión de la época derivada del estudio de este yacimiento no contradice realmente el modelo general sino que descubre sus fallos en la reconstrucción histórica y lo complementa y corrige. Por todo ello se puede considerar que el hábitat de Portecelo es la otra cara hasta ahora olvidada o minusvalorada de la Edad del Bronce y al igual que en una moneda existen cara y cruz, las dos visiones que hemos manejado, la general canónica y la particular del yacimiento, son inseparables, complementarias y en modo alguno excluyentes, sino que, por el contrario, el contraste de las dos dará una visión más completa y rigurosa del Bronce Final del noroeste de la Península Ibérica. Los estudios actualmente en curso, datación absoluta por C14, reconstrucción de la paleoecología del entorno, la integración de Portecelo en el ámbito regional y otros, permitirán precisar mucho más la función y significado del yacimiento en el contexto geográfico y aquilatar más la reconstrucción histórica de la Edad del Bronce en la zona en particular y en la fachada atlántica de la Península Ibérica en general. 
El término «complejo técnico de cantos tallados", un tipo particular, del concepto "complejo técnicon, ha sido un instrumento útil para describir realidades arqueológicas poco encuadrables con rigor dentro de los más tradicionales y equivocos de cultura o civilización. Este instrumento conceptual ha sido usado con eficacia para evitar generalizaciones tan peligrosas como "cultura achelense" o "cultura de los cantos tallados" pero poco a poco ha ido adquiriendo tal polisemia que su valor actual en algunos autores es demasiado genérico y por ello escasamente operativo ya que llega a alcanzar, de hecho, el significado tan amplio de conjunto de productos líticos obtenidos a partir de cantos. Pero como muestra la comparación de la industria de Portecelo con los materiales de yacimientos próximos, de época Paleolítica o de la Edad del Hierro, hay una serie de rasgos comunes claros entre ellos, al lado de unas diferencias que se derivan básicamente de su naturaleza cultural. Por ello el empleo del término «complejo técnico de los cantos tallados" se presenta como demasiado vago y confuso y en tanto en cuanto contribuye más a mermar la significación de los conjuntos líticos singulares de los diferentes yacimientos que a ser un concepto operativo que ayude a clarificar la naturaleza de las industrias líticas y por ende de la actividad humana, debe ser cuidadosamente manejado o, en su caso, su empleo ha de ser muy restringido. Trabajos en curso sobre el tema permitirán mayores precisiones sobre el concepto de «complejo técnico de cantos tallados" y su problemática actual.

\section{BIBLIOGRAFIA}

Briard, J. (1985): L'Age du Bronce en Europe. París.

CANO, J. (1987): «La industria lítica». En: Carballo, X.: Castro da Forca. Campaña 1984. Arqueoloxia/Memorias, 8, Santiago.

- (1988a): „El yacimiento de Portecelo (O Rosal, Pontevedra)». Pontevedra Arqueológica, 3, Pontevedra.

- (1988b): Las industrias líticas talladas en la costa de La Guardia a Baiona (Pontevedra). La Coruña.

- (1988c): "La industria lítica tallada en la Cultura Castreña de Galicia», I Congreso Peninsular de Historia Antigua. Santiago.

PENA, A. (1987): «Tres años de excavaciones arqueológicas en el yacimiento galaico-romano de Santa Trega (A Guarda, Pontevedra: 1983-1985». Pontevedra Arqueológica, 2, Pontevedra.

QUEROL, M., y SANTONJA, M. (1978): «Sistema de clasificación de cantos trabajados y su aplicación en yacimientos del Paleolítico Antiguo de la Península Ibéricaw. Saguntum, 13, Valencia.

VIDAL, J. (1983): «El yacimiento achelense de las Gándaras de Budiño: La colecciọn Pelayo Fernández. Estudio tipológico de bifaces, cantos trabajados y hendedores". II Seminario de Arqueología del Noroeste. Santiago, 1980. Madrid. 